\title{
Changes in Inpatient Surgery Utilization at the Community Level
}

\author{
Ronald Lagoe*, Mark Murphy and Shelly Littau \\ Hospital Executive Council, Syracuse, New York, 13235, USA \\ *Corresponding author: Ronald Lagoe, Hospital Executive Council, Syracuse, New York, 13235, USA \\ To Cite This Article: Ronald Lagoe, Shelly Littau, Changes in Inpatient Surgery Utilization at the Community Level. Am J Biomed Sci \& Res. 2021 \\ - 12(3). AJBSR.MS.ID.001751. DOI: 10.34297/AJBSR.2021.12.001751.
}

Received: 眥 February 02, 2021; Published: 畊 March 31, 2021

\section{Opinion}

Historically, health care utilization in the United States has changed slowly. This has been especially true for hospital utilization at the community wide level.

The utilization of hospitals and other health care providers is usually based on large numbers of patients. Changes in the characteristics of these patients occur over long periods of time.

This brief study summarized changes in adult surgery discharges by month in the metropolitan area of Syracuse, New York between 2018 and 2020. During this period, hospital utilization was affected by at least two important developments that occurred in recent years.

One of these was the movement of procedures involving relatively healthy patients from inpatient to outpatient status. Examples of these patients included those individuals receiving joint replacements who had small numbers of comorbidities and could be treated on an outpatient basis.

Another development was the onset of the Coronavirus epidemic in the United States during 2020. This epidemic resulted in the postponement of large numbers of elective surgeries in order to make room for adult medicine patients with respiratory diagnoses.

This study focused on inpatient utilization in the hospitals of Syracuse, New York for both of these issues between 2018 and 2020. The Syracuse hospitals treated 21,701 inpatient adult surgery discharges during 2018, 21,429 discharges during 2019, and 17,977 discharges during 2020. The distribution of these discharges during 2020 included 3,803 patients at Crouse Hospital, 7,188 patients at St. Joseph's Hospital Health Center, and 6,986 patients at Upstate University Hospital.

The study data indicated that inpatient orthopedic surgery discharges in the combined Syracuse hospitals declined from 8,175 in January - December 2018 to 7,835 in 2019, and 5,929 in 2020. The decline in orthopedic surgery discharges between 2018 and 2019 and a portion of the decline between 2019 and 2020 was generated by the movement of individuals from inpatient hospitals to outpatient surgery centers. This decline was distributed relatively evenly over the twelve-month periods.

Table 1: Inpatient Adult Surgery Hospital Discharges by Month Syracuse Hospitals.

\begin{tabular}{|c|c|c|c|c|c|c|c|c|c|c|c|c|}
\hline \multicolumn{13}{|c|}{ January - December 2018 - 2020} \\
\hline & Jan & Feb & Mar & Apr & May & Jun & Jul & Aug & Sept & Oct & Nov & Dec \\
\hline 2018 & 1,756 & 1,689 & 1,853 & 1,747 & 1,906 & 1,844 & 1,746 & 1,893 & 1,812 & 1,940 & 1,745 & 1,770 \\
\hline 2019 & 1,774 & 1,712 & 1,791 & 1,769 & 1,926 & 1,679 & 1,817 & 1,856 & 1,724 & 1,922 & 1,757 & 1,702 \\
\hline 2020 & 1,752 & 1,656 & 1,494 & 830 & 1,012 & 1,552 & 1,751 & 1,638 & 1,729 & 1,732 & 1,527 & 1,304 \\
\hline Difference $2018-2020$ & -4 & -33 & -359 & -917 & -894 & -292 & 5 & -255 & -83 & -208 & -218 & -466 \\
\hline Difference 2019-2020 & -22 & -56 & -297 & -939 & -914 & -127 & -66 & -218 & 5 & -190 & -230 & -398 \\
\hline Percent Difference 2018-2020 & -0.2 & -2 & -19.4 & -52.5 & -46.9 & -15.8 & 0.3 & -13.5 & -4.6 & -10.7 & -12.5 & -26.3 \\
\hline
\end{tabular}

Adult surgery data exclude Diagnosis Related Groups concerning medicine, obstetrics, psychiatry, alcohol / substance abuse treatment, and all patients aged $0-17$ years.

Source: Hospital Executive Council. 
The study data also indicated that much of the decline focused on specific monthly periods. These data are summarized in the table 1 which follows.

The data in the study table identify small reductions in adult surgery discharges between January - February 2020, before the onset of the Coronavirus epidemic. These declines were followed by reductions of 26 - 73 percent between April - June 2020, during the initial outbreak of the epidemic. The reductions in adult surgery declined between July - October 2020 as the outbreak subsided. Another increase followed in December 2020.

The changes in adult surgery volumes in the Syracuse hospitals during 2020 demonstrated the size of the impact of the Coronavirus at the community level. These data are probably typical of the impact of the epidemic in other communities.

The experiences of adult surgery utilization in the Syracuse hospitals during the past three years outline different types of situations that have addressed surgery utilization at the community level. The movement of patients from inpatient to outpatient settings has been generated by the capabilities of different providers and related financial issues. The Coronavirus epidemic is a widespread epidemiological issue that has affected large populations.

In both situations, surgery utilization has been in the position of reacting to economic and clinical developments at the community level. The data in this study suggest that surgery has been on the receiving end of these developments.

In the future, surgery and related health care specialties need to consider additional planning approaches in order to respond to these developments. Such approaches should involve addressing them in a proactive manner. 